\title{
$\Omega$
}

[1]

12nd Dept of Internal Medicine, University Hospital of Giessen and Marburg, Giessen, Germany.

2Dept of Thoracic Medicine, Interstitial Lung Disease Unit and Laboratory of Molecular and Cellular

Pneumonology, Medical School, University of Crete, Heraklion, Greece.

${ }^{3}$ Oxford Centre fore Respiratory Medicine and Oxford Respiratory Trials Unit, Oxford University Hospitals NHS

Foundation Trust, Oxford, UK.

${ }^{4}$ Laboratory of Pleural and Lung Cancer Translational Research, Nuffield Department of Medicine, University of

Oxford, UK.

ioannis.psallidas@ndm.ox.ac.uk

in

https://www.linkedin.com/in/ ioannis-psallidas-7a4258a6/

\section{Respiratory training in Greece}

In Greece, respiratory medicine is a stand-alone specialty requiring a 5 -year training programme. Acquisition of the general medicine specialty is not a prerequisite for entering the respiratory training programme. As background information, Greece's population is approximately 11 million and there are tertiary hospitals covering specific geographical areas of the country. The majority of the tertiary hospitals include respiratory wards and also train registrars. Although there is heterogeneity between different hospitals, our article highlights the training of respiratory physicians in the majority of respiratory departments in the country.

\section{Training programme}

To enter a respiratory medicine programme in Greece, junior doctors must register on their desired hospital's list. Training is provided in public, university and military hospitals. Training in outpatient clinics outside the accredited hospitals or in private practices is not possible, in contrast to other countries such as Germany. The mean waiting period is 2 years for high-quality, popular university hospitals. In smaller hospitals, however, it is possible that there is no waiting time to enter the training programme.

There are still two major hospitals in Athens and Thessaloniki for the treatment of respiratory patients, initially built as "sanatoria" during the tuberculosis era. The trend, however, is that in every general hospital, there is a department for respiratory medicine, while the two large specialist hospitals for respiratory medicine have evolved to become tertiary hospitals.

Respiratory medicine is a run-through specialty. The 5-year training programme involves a 1-year period of training in internal medicine and a 6-month period training in intensive care medicine (ICM). The training in specific areas of expertise varies from hospital to hospital, given that some hospitals lack certain subspecialties, such as managing lung cancer patients with chemotherapy, cystic fibrosis units and interventional procedures (e.g. thoracoscopy or rigid bronchoscopy). The training programme mostly involves in- and outpatient care and education in practical procedures and interventions, such as chest drain insertion, flexible bronchoscopy and thoracic ultrasound. The emerging field of medical thoracoscopy is not available in every hospital in Greece but there is a growing number of respiratory physicians who are trained abroad and transfer the knowledge back to their home institutions.

The training programme is a full-time job, meaning that job sharing or working $50 \%$ of the time is not possible, with the exception of colleagues who become pregnant or take some time off after giving birth. At the end of the programme, trainees must pass written and oral examinations in front of a three-member committee of certified pulmonologists to receive their licence to practice.

\section{Life as a respiratory trainee}

Initially, respiratory registrars are trained mainly on the respiratory wards and each trainee is assigned to a consultant for a minimum 6-month period. There is also a strict learning programme covering every aspect of respiratory medicine and registrars are expected to follow the required curriculum. All registrars are trained in bronchoscopy and based on each department, expertise in additional interventional procedures (pleural diseases, endobronchial ultrasound-guided bronchoscopy and interventional bronchoscopy). They are also expected to develop teaching and presentation skills though one-to-one teaching of medical students or junior colleagues and presenting interesting cases in grand rounds. At a later stage of their training, registrars are involved more in respiratory outpatient management.

When on call, trainees are responsible for approximately 40 inpatients in the respiratory ward. Working hours vary between hospitals but mostly, shifts start at 08:00 h and finish around $18: 00 \mathrm{~h}$. For 7 days each month, the interns are on call, meaning a 24-h shift that sometimes turns into
Cite as: Drakopanagiotakis F, Trachalaki A, Psallidas I. Respiratory training in Greece. Breathe 2017; 13: 251-253. 


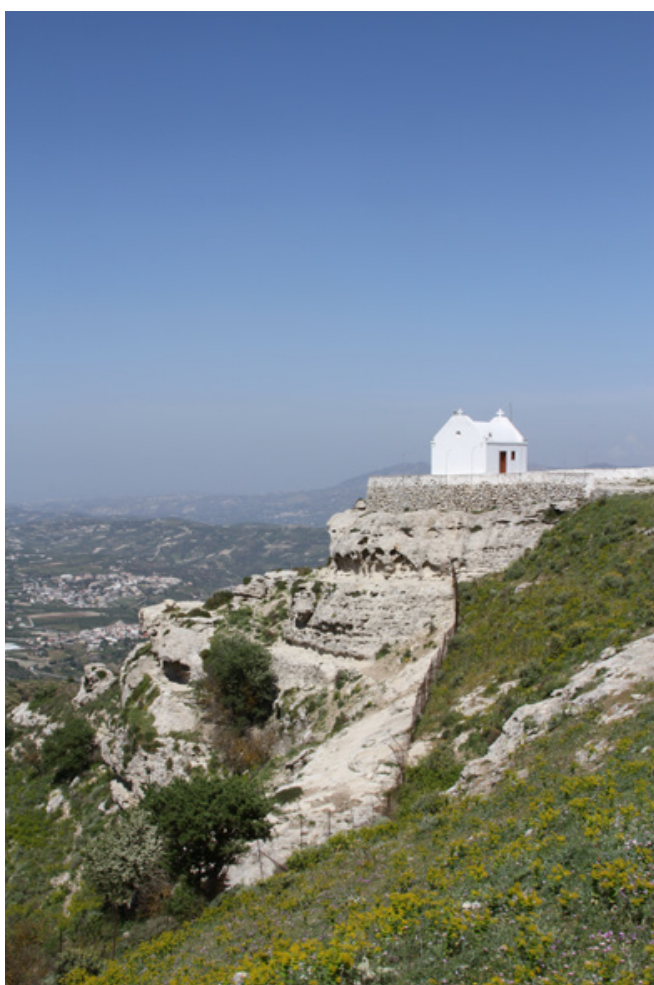

an exhausting 32-h shift due to lack of personnel and workload. Additionally, trainees support medical and surgical wards by covering the hospital referrals in collaboration with a consultant.

Trainees also cover the shifts for respiratory patients seen in the emergency department. Together with the respiratory consultant, they decide about submissions and further treatment. In smaller hospitals, it is possible that interns in respiratory medicine share shifts in the emergency department with their colleagues in general internal medicine.

Continuous medical education (CME) is actively supported in Greece, and there are many seminars and courses to help physicians improve their skills. Although a certain number of CME credits is not a formal requisite for re-accreditation, the majority of pulmonologists easily reach the 150-credit limit in a 5-year period. For many years, the Hellenic Respiratory Society has endorsed the European Respiratory Society Harmonised Education in Respiratory Medicine for European Specialists (HERMES) Curriculum and supports its members through courses in order to take the HERMES Examination in Adult Respiratory Medicine.

A typical day at work for a first-year registrar starts at about 08:00 h. For $1 \mathrm{~h}$, we have a meeting with the respiratory team (including consultants, registrars and medical students) to discuss all inpatients, and arrange duties and tasks that need to be completed within the day. Specifically, I have to review my patients first, inform the consultant for their progress, attend the ward round and prepare the discharges in due course. Additionally, alongside a consultant, I review all the new patients that are transferred to the ward. If I am on call at 16:00 h, I move to the Accident and Emergency department to review respiratory patients and decide, with my consultant, on their care.

\section{Research perspective}

Some registrars decide early in their career or before specialisation an academic orientation by pursuing a PhD degree. In a university hospital in Greece, medical students and registrars have plenty of opportunities to get involved in research and contribute to medical advancements in respiratory medicine. There is a number of registrars who combine clinical and academic training in respiratory medicine.

\section{Career as a respiratory consultant}

As respiratory medicine specialists, physicians can work either in public hospitals and outpatient clinics or in private practices and clinics. Getting a job in a public hospital (either a district or university hospital) as a specialist is a highly competitive procedure. The procedure itself lasts a significant period of time, reaching up to a couple of years, due to the centralised procedure, demanding the complete overview of the procedure from the Greek Ministry of Health. Consultants are responsible for respiratory inpatients and for those seen in the emergency department. This requires five to seven shifts per month during which the consultant is required to be physically present in the hospital. Consultants perform interventional procedures, and are responsible for the teaching and training of students, registrars and fellows. In tertiary hospitals, it is expected that they actively participate in audit and research programmes, and can show a respectable research record, in order to achieve promotion or sometimes to remain their job.

Many respiratory physicians pursue a career in ICM. ICM training requires an extra 2 years of training after completing the basic training. Despite the obvious challenges in ICM, many physicians choose to follow this path, as there is a high demand for ICM specialists in Greece.

A significant number of respiratory consultants decide to work in a private practice. The work can be especially challenging but rewarding as well, taking into account the potential for primary and secondary prevention. Specifically, smoking cessation services, student education in schools and public presentations concerning respiratory disease are often initiated by colleagues in private practice.

\section{Challenges due to the financial crisis}

Due to the financial crisis in recent years, many medical school graduates have chosen to enter a 
training programme abroad rather than remain in Greece. This has subsequently led to a decrease in the number of respiratory registrars in Greece. Even major teaching hospitals face difficulties in recruiting trainees. In addition, a significant number of respiratory consultants have decided to work abroad due to salary cuts, decreased recruitment in hospitals and financial difficulties in maintaining a private practice. Hospitals, universities and professional societies, however, actively maintain and develop the level of respiratory medicine through collaborative projects between different institutions, and with many of their alumni and residents working abroad.

\section{Conclusion}

Respiratory medicine is a challenging specialty that requires a combination of theoretical knowledge and practical skills. It is a rewarding specialty that has a great impact in improving respiratory patient care, and promoting collaboration among physicians, team building and support.

\section{Support statement}

I. Psallidas is the recipient of a REPSIRE2 European Respiratory Society Fellowship (grant number 2015-7160). Funding information for this article has been deposited with the Crossref Funder Registry.

\section{Conflict of interest}

None declared. 